\title{
Inverse problem for non-stationary system of magnetohydrodynamics
}

\author{
Undasin U Abylkairov and Serik E Aitzhanov ${ }^{*}$
}

${ }^{*}$ Correspondence:

SAitzhanov@mail.ru

Department of Differential

Equations and Control Theory,

Kazakh National University Named

of Al-Farabi, Al-Farabi 71, Almaty,

050038, Kazakhstan

\begin{abstract}
We study the inverse problem for non-stationary system of magnetic hydrodynamics in which it is required to determine the velocity of the fluid $\vec{v}(x, t)$, the magnetic tension $\vec{H}(x, t)$, the pressure gradient $\nabla p(x, t)$, but also the external forces $\vec{f}(x)$ and the current $\operatorname{rot} \vec{j}(x)$. In this case, to the conditions constituting the direct problem are added additional conditions. The trace speed, the magnetic tension, and the pressure gradient in the final moment, time $t=T$, are taken as additional information. The strong generalized solvability of the inverse problem in the two-dimensional case is proved.
\end{abstract}

Keywords: magnetohydrodynamics; inverse problem; final overdetermination condition

\section{Introduction}

The mathematical description of the processes occurring in moving fluids leads to the solution of the Navier-Stokes equations. References [1-8] are devoted to the study of the questions of solvability and stability of solutions of initial-boundary value problems for the linearized and general nonlinear Navier-Stokes equations.

Magnetohydrodynamics (MHD) is a theory of macroscopic interaction of electrically conductive fluid and electromagnetic fields. It has important applications in astronomy and geophysics, as well as in engineering fields such as controlled thermonuclear fusion, nuclear reactor cooling liquid metals, electromagnetic casting of metals, MHD generators, and MHD ion engines.

In the 1960s and 1970s the efforts of mathematicians were directed to the study of a class of problems of magnetohydrodynamics. Fundamental work in this direction was done by Ladyzhenskaya and Solonnikov $[9,10]$.

In this paper we study the three initial-boundary value problems for non-stationary magnetohydrodynamic equations. Results on the solvability of these problems are similar to the corresponding results on the solvability of the initial-boundary value problems for non-stationary Navier-Stokes equations. Similar results were obtained by Mosconi and Solonnikov in [11] for stationary MHD equations. A variety of approaches to the mathematical study of MHD systems are reflected in the work of Ladyzhenskaya and Solonnikov [12], Sahaev and Solonnikov [13], Stupyalis [14, 15], Alekseev and Tereshko [16], Duvant and Lions [17], Sermange and Temam [18], Giga and Yoshida [19], and Dyer and Edmuns [20]. 


\section{Statement of the problem}

We consider the inverse problem of magnetic hydrodynamic in the cylinder $Q_{T}=\Omega \times$ $[0, T], \Omega \subset R^{2}$. One can take the border of the area $\Omega$ from $C^{2}$, namely $\partial \Omega \subset C^{2}, \Gamma=$ $\partial \Omega \times[0, T]$. We need to determine $\vec{v}(x, t), \vec{H}(x, t), \nabla p(x, t), \vec{f}(x)$, and rot $\vec{j}(x)$ that satisfy the following equations:

$$
\begin{aligned}
& \frac{\partial \vec{v}}{\partial t}+\sum_{k=1}^{2} v_{k} \vec{v}_{x_{k}}-\frac{\mu}{\rho} \sum_{k=1}^{2} H_{k} \vec{H}_{x_{k}}-v \Delta \vec{v}=-\frac{1}{\rho} \operatorname{grad}\left(p+\frac{\mu \vec{H}^{2}}{2}\right)+g(x, t) \vec{f}(x), \\
& \frac{\partial \vec{H}}{\partial t}+\frac{1}{\sigma \mu} \operatorname{rot} \operatorname{rot} \vec{H}-\operatorname{rot}[\vec{v} \times \vec{H}]=\frac{\xi(x, t)}{\sigma \mu} \operatorname{rot} \vec{j}(x), \\
& \operatorname{div} \vec{v}=0, \quad \operatorname{div}(\mu \vec{H})=0 .
\end{aligned}
$$

We have the initial conditions

$$
\vec{v}(x, 0)=\vec{v}_{0}(x), \quad \vec{H}(x, 0)=\vec{H}_{0}(x),
$$

the boundary conditions

$$
\left.\vec{v}\right|_{\Gamma}=0,\left.\quad H_{n}\right|_{\Gamma}=0, \quad \frac{\partial H_{2}}{\partial x_{1}}-\left.\frac{\partial H_{1}}{\partial x_{2}}\right|_{\Gamma}=\left.j\right|_{\Gamma}=0
$$

and the overdetermination conditions

$$
\vec{v}(x, T)=\vec{U}(x), \quad \vec{H}(x, T)=\vec{\Psi}(x), \quad \nabla p(x, T)=\nabla \pi(x) .
$$

Here $\vec{v}(x, t)$ is the velocity of the fluid, $\vec{H}(x, t)$ the magnetic tension, $p$ the pressure, $g(x, t) \vec{f}(x)$ the external hydrodynamic forces, $\xi(x, t) \operatorname{rot} \vec{j}(x)$ the current, $\mu$ the magnetic permeability, $\sigma$ the conductivity, $\rho$ the condensation, $v$ the kinematic viscosity coefficient of the fluid, $\vec{n}$ the outward pointing normal vector to the surface area $S$, and $H_{n}=\vec{H} \cdot \vec{n}$.

The first results of the well-posedness of the inverse problem with homogeneous boundary conditions for the Navier-Stokes equations appeared in the works of Prilepko and Vasin [21] and Abylkairov [22]. Reference [23] studied the solvability of the inverse spatial problem with unknown right part where we obtain a local existence theorem for the solutions. Different inverse problems for the Navier-Stokes equations and hydrodynamics were presented in [24-35]. In the work of Abylkairov [30] the inverse problem for the Navier-Stokes equations was studied with non-standard boundary conditions. The controllability of the systems of magnetic hydrodynamics has been studied in many papers (see, for example, [16, 31-35]).

We denote by $J_{1}(\Omega)$ the subspace $\stackrel{0}{W}_{2}^{1}(\Omega)$, consisting of solenoidal vectors and by $\hat{J}(\Omega)$ the subspace $W_{2}^{2}(\Omega)$, consisting of solenoidal vectors, satisfying the following $\Gamma=\partial \Omega \times[0, T]$ conditions: $\left.u_{n}\right|_{\Gamma}=0,\left.y \operatorname{rot} \vec{u}\right|_{\Gamma}=0$. Let $V_{2}\left(Q_{T}\right)$ be the Banach space of functions with the norm $\|\vec{u}\|_{V_{2}\left(Q_{T}\right)}=\operatorname{vrai}_{\max _{0 \leq t \leq T}}\|\vec{u}(x, t)\|_{2, \Omega}+\left\|\vec{u}_{x}\right\|_{2, Q_{T}}$ that is obtained as a result of the closure of set of smooth, solenoidal, and zero vectors near $\Gamma=\partial \Omega \times[0, T] . \stackrel{0}{J}_{l, n}(\Omega)$ is a subset of $W_{2}^{l}(\Omega)$ ( $l$ is an integer number), for which the closure in the norm of $W_{2}^{l}(\Omega)$ of the set of continuously differentiable solenoidal vector-functions with $\left.u_{n}\right|_{\Gamma}=0 . G(\Omega)$ 
consists of $\operatorname{grad} \varphi$, where $\varphi$ is a single-valued function in $\Omega$, a locally square integrable and differentiable function in $L_{2}(\Omega)$.

Lemma 1 ([4]) The following inequalities hold:

$$
\|u\|_{4, \Omega}^{4} \leq 4\|u\|_{2, \Omega}^{2}\left\|u_{x_{1}}\right\|_{2, \Omega}\left\|u_{x_{2}}\right\|_{2, \Omega} \leq 2\|u\|_{2, \Omega}^{2}\left\|u_{x}\right\|_{2, \Omega}^{2}
$$

for all $u(x) \in \stackrel{0}{W}_{2}^{1}(\Omega), \Omega \subset R^{2}$,

$$
\|u\|_{4, \Omega}^{4} \leq \varepsilon\left\|u_{x}\right\|_{2, \Omega}^{4}+\varepsilon^{-1}\|u\|_{2, \Omega}^{4}
$$

where $\varepsilon$ is optional $(\varepsilon>0)$,

$$
\begin{aligned}
& \|u\|_{4, \Omega} \equiv\left(\int_{\Omega}|u|^{4} d x\right)^{\frac{1}{4}}, \quad\left|u_{x}\right| \equiv\left(\sum_{k=1}^{2} u_{x_{k}}^{2}\right)^{\frac{1}{2}}, \\
& \left\|u_{x}\right\|_{2, \Omega}^{2} \equiv \int_{\Omega}\left|u_{x}\right|^{2} d x \equiv \int_{\Omega} u_{x}^{2} d x .
\end{aligned}
$$

Theorem 1 ([15]) If $\partial \Omega \in C^{2}$, then the operator rot sets a one-to-one correspondence between the spaces $W_{2}^{1}(\Omega)$ and ${ }^{0}(\Omega)$, moreover, the following inequality holds:

$$
\|\operatorname{rot} \vec{V}\| \leq\|\vec{V}\|_{2, \Omega}^{(1)} \leq\left(\sqrt{2}+\frac{1}{\sqrt{\mu_{1}}}\right)\|\operatorname{rot} \vec{V}\|
$$

for all $\vec{V} \in \stackrel{0}{W}_{2}^{1}(\Omega)$.

Here the number $\mu_{1}$ is the smallest eigenvalue of the operator $\Delta$ in the field $\Omega$ at the zero boundary condition.

Theorem 2 ([15]) If $\partial \Omega \in C^{2}$, then the operator rot gives a one-to-one map of $J_{1, n}^{0}(\Omega)$ onto $L_{2}(\Omega)$, moreover, the following inequality holds:

$$
\|\operatorname{rot} \vec{\psi}\| \leq\|\vec{\psi}\|_{2, \Omega}^{(1)} \leq C_{3}\|\operatorname{rot} \vec{\psi}\|
$$

for all $\vec{\psi}=\left(\psi_{1}, \psi_{2}\right) \in \stackrel{0}{J}_{1, n}(\Omega)$. Here $\vec{\psi}$ does not depend on the constant $C_{3}$.

\section{Main results}

Definition 1 The generalized solution of the inverse problem (1)-(6) is a set of function $\{\vec{v}(x, t), \nabla p(x, t), \vec{H}(x, t), \vec{f}(x), \operatorname{rot} \vec{j}(x)\}$, satisfying the correspondence (1)-(6) in the case if $\vec{v}(x, t) \in W_{2}^{2,1}\left(Q_{T}\right) \cap \stackrel{0}{J}_{1}\left(Q_{T}\right), \vec{H}(x, t) \in W_{2}^{2,1}\left(Q_{T}\right) \cap \hat{J}\left(Q_{T}\right), \vec{f}(x) \in L_{2}(\Omega)$, rot $\vec{j}(x) \in L_{2}(\Omega)$, and the function $p(x, t) \in G\left(Q_{T}\right)\left(\nabla p \in L_{2}\left(Q_{T}\right)\right)$ at any $t$ from $[0, T]$, and it continuously depends on $t$ in the norm of this space for $[0, T]$.

Let us fix the functions $g(x, t)$ and $\xi(x, t)$ and define the nonlinear operators $T_{g}: L_{2}(\Omega) \rightarrow$ $L_{2}(\Omega), S_{\xi}: L_{2}(\Omega) \rightarrow L_{2}(\Omega)$ by the following expression:

$$
\left(T_{g} \vec{f}\right)(x)=\vec{v}_{t}(x, T), \quad\left(S_{\xi} \vec{r}\right)(x)=\vec{H}_{t}(x, T)
$$


Here $r=\operatorname{rot} \vec{j}(x), \vec{f}=\vec{f}(x)$, but $\vec{v}(x, t)$ and $\vec{H}(x, t)$ are the solution of the direct problem (1)-(5) with $\vec{F}=g(x, t) \vec{f}(x), \operatorname{rot} \vec{J}_{0}=\xi(x, t) \operatorname{rot} \vec{j}(x)$.

The introduced operators $T_{g}$ and $S_{\xi}$ were well-posed as regards their definitions, since the necessary conditions for differentiability of $\vec{v}, \vec{H}$, and $p$ are ensured by the theory in the work of Ladyzhenskaya and Solonnikov (see [9] Theorem 18, p.168, Theorem 19, p.169).

We suppose that $g(x, T) \neq 0$ and $\xi(x, T) \neq 0$ for all $x \in \Omega$; we introduce the nonlinear operators $A: L_{2}(\Omega) \rightarrow L_{2}(\Omega)$ and $y B: L_{2}(\Omega) \rightarrow L_{2}(\Omega)$, by the following expressions:

$$
(A \vec{f})(x)=\frac{1}{g(x, T)}\left(T_{g} \vec{f}\right)(x), \quad(B \vec{r})(x)=\frac{\sigma \mu}{\xi(x, T)}\left(S_{\xi} \vec{r}\right)(x) .
$$

Thus, if $g(x, t) \vec{f}(x) \in L_{2}\left(Q_{T}\right), g_{t}(x, t) \vec{f}(x) \in L_{2,1}\left(Q_{T}\right)$ and $\xi(x, t) \operatorname{rot} \vec{j}(x) \in L_{2}\left(Q_{T}\right), \xi_{t}(x, t) \times$ $\operatorname{rot} \vec{j}(x) \in L_{2,1}\left(Q_{T}\right)$, additionally $g(x, t), g_{t}(x, t) \in C\left(\bar{Q}_{T}\right), \xi(x, t), \xi_{t}(x, t) \in C\left(\bar{Q}_{T}\right)$, then, by (1) and (2) and in terms of these operators, (7), (8) given the inverse problem can be rewritten as

$$
A \vec{f}+\vec{\kappa}=\vec{f}, \quad B \vec{r}+\vec{\lambda}=\vec{r}
$$

where

$$
\begin{aligned}
& \vec{\aleph}=\frac{1}{g(x, T)}\left[-v \Delta \vec{U}+U_{k} \vec{U}_{x_{k}}-\frac{\mu}{\rho} \Psi_{k} \vec{\Psi}_{x_{k}}+\frac{1}{\rho} \nabla\left(\pi+\frac{\mu \vec{\Psi}^{2}}{2}\right)\right], \\
& \vec{\lambda}=\frac{\sigma \mu}{\xi(x, T)}\left[\frac{1}{\sigma \mu} \operatorname{rot} \operatorname{rot} \vec{h}-\operatorname{rot}(\vec{U} \times \vec{\Psi})\right] .
\end{aligned}
$$

Theorem 3 Assume that $\Omega \subset R^{2}, g, g_{t} \in C\left(\bar{Q}_{T}\right), \xi, \xi_{t} \in C\left(\bar{Q}_{T}\right),|g(x, t)| \geq g_{T}>0,|\xi(x, t)| \geq$ $\xi_{T}>0$ for $x \in \Omega, \vec{U}(x) \in W_{2}^{2}(\Omega) \cap J_{1}(\Omega), \vec{H}_{0}(x) \in \hat{J}(\Omega), \vec{\Psi}(x) \in \hat{J}(\Omega), \vec{v}_{0}(x) \in W_{2}^{2}(\Omega) \cap J_{1}(\Omega)$, $\nabla \pi(x) \in G(\Omega)$. Then the operators $A$ and $B$ are completely continuous from $L_{2}(\Omega)$ to $L_{2}(\Omega)$.

Proof Now we show that the operators $T_{g}$ and $S_{\xi}$ are completely continuous. Assume that $\vec{f}(x)$ and $\vec{r}(x)$ are arbitrary elements of $L_{2}(\Omega)$. We take arbitrary sequences $\left\{\vec{f}^{N}\right\}$ and $\left\{\vec{r}^{N}\right\}$ of $L_{2}(\Omega)$, such that

$$
\left\|\vec{f}^{N}-\vec{f}\right\| \rightarrow 0, \quad\left\|\vec{r}^{N}-\vec{r}\right\| \rightarrow 0, \quad \text { as } N \rightarrow \infty
$$

Let us show (10) implies that

$$
\left\|T_{g} \vec{f}^{N}-T_{g} \vec{f}\right\| \rightarrow 0, \quad\left\|S_{\xi} \vec{r}^{N}-S_{\xi} \vec{r}\right\| \rightarrow 0, \quad \text { as } N \rightarrow \infty .
$$

We consider in $Q_{T}$ the following problem:

$$
\begin{aligned}
& \vec{W}_{t}-v \Delta \vec{W}+v_{k}^{N} \vec{W}_{x_{k}}-\frac{\mu}{\rho} H_{k}^{N} \vec{h}_{x_{k}}=-\frac{1}{\rho} \nabla\left(p+\frac{\mu\left(\vec{H}^{N}-\vec{H}\right)^{2}}{2}\right)+\vec{F} \\
& \vec{h}_{t}+\frac{1}{\sigma \mu} \operatorname{rot} \operatorname{rot} \vec{h}-H_{k}^{N} \vec{W}_{x_{k}}+v_{k}^{N} \vec{h}_{x_{k}}=\vec{G}, \\
& \operatorname{div} \vec{W}=0, \quad \operatorname{div} \vec{h}=0,
\end{aligned}
$$




$$
\begin{array}{ll}
\left.h_{n}\right|_{\Gamma}=0, & \left.\operatorname{rot}_{\tau} \vec{h}\right|_{\Gamma}=0,\left.\quad \vec{W}\right|_{\Gamma}=0, \\
\left.\vec{W}\right|_{t=0}=0, & \left.\vec{h}\right|_{t=0}=0 .
\end{array}
$$

Here we introduce the notations $\vec{W}=\vec{v}^{N}-\vec{v}, \vec{h}=\vec{H}^{N}-\vec{H}, \vec{F}=\left(\vec{f}^{N}-\vec{f}\right) g-W_{k} \vec{v}_{x_{k}}+\frac{\mu}{\rho} h_{k} \vec{H}_{x_{k}}$, $\vec{G}=\frac{\xi(x, t)}{\sigma \mu}\left(\vec{r}^{N}-\vec{r}\right)+h_{k} \vec{v}_{x_{k}}-W_{k} \vec{H}_{x_{k}}$. Here the functions $\vec{v}^{N}, \vec{H}^{N}$, and $\nabla p^{N}$ are the generalized solution of the direct problem (1)-(5), corresponding to the external forces $\vec{f}^{N}(x) g(x, t)$ and the currents $\xi(x, t) \vec{r}^{N}(x)$. We denote the solution of this problem, corresponding to the external forces $\vec{f}(x) g(x, t)$ and the currents $\xi(x, t) \vec{r}(x)$, by $\vec{v}, \vec{H}$, and $\nabla p$.

We can consider the problem (14)-(18) with respect to the functions $\vec{W}$ and $\vec{h}$ as linear, since $(\vec{v}, \vec{H})$ and $\left(\vec{v}^{N}, \vec{H}^{N}\right)$ have the following $[9,13-15]$ differential conditions: $\left(\vec{v}_{t x}, \vec{H}_{t x}\right) \in$ $L_{2}\left(Q_{T}\right) \times L_{2}\left(Q_{T}\right)$ and $\left(\vec{v}_{t x}^{N}, \vec{H}_{t x}^{N}\right) \in L_{2}\left(Q_{T}\right) \times L_{2}\left(Q_{T}\right)$; then $(\vec{v}, \vec{H})$ and $\left(\vec{v}^{N}, \vec{H}^{N}\right)$ are elements $L_{q}(\Omega) \times L_{q}(\Omega)$, for all $t \in[0, T]$ with any finite $q$, and they continuously depend on $t$ in the norm $L_{q}(\Omega) \times L_{q}(\Omega)$. The following inequality holds:

$$
\begin{aligned}
& \left\|\vec{W}_{t}\right\|_{2, Q_{T}}^{2}+\left\|\vec{h}_{t}\right\|_{2, Q_{T}}^{2}+v\left\|\vec{W}_{x}\right\|_{2, \Omega}^{2}+\frac{1}{\sigma \mu}\left\|\vec{h}_{x}\right\|_{2, \Omega}^{2} \\
& \quad+v\|P \Delta \vec{W}\|_{2, Q_{T}}^{2}+\frac{1}{\sigma \mu}\|\operatorname{rot} \operatorname{rot} \vec{h}\|_{2, Q_{T}}^{2} \leq c\left(\|\vec{F}\|_{2, Q_{T}}^{2}+\|\vec{G}\|_{2, Q_{T}}^{2}\right)
\end{aligned}
$$

for the solution of the problem $[8,9,13]$.

Let us estimate $\|\vec{F}\|_{2, Q_{T}}^{2}$ and $\|\vec{G}\|_{2, Q_{T}}^{2}$. By applying Theorems 1 and 2, and Lemma 1, we obtain

$$
\begin{aligned}
\|\vec{F}\|_{2, Q_{T}}^{2} \leq 3\left[\left\|\left(\vec{f}^{N}-\vec{f}\right) g\right\|_{2, Q_{T}}^{2}+\left\|W_{k} \vec{v}_{x_{k}}\right\|_{2, Q_{T}}^{2}+\frac{\mu}{\rho}\left\|h_{k} \vec{H}_{x_{k}}\right\|_{2, Q_{t}}^{2}\right] \\
\left\|W_{k} \vec{v}_{x_{k}}\right\|_{2, Q_{T}}^{2}=\left\|\left(\vec{v}^{N}-\vec{v}\right)_{k} \cdot \vec{v}_{x_{k}}\right\|_{2, Q_{T}}^{2}=\int_{0}^{T} \int_{\Omega}\left(\vec{v}^{N}-\vec{v}\right)_{k}^{2} \cdot \vec{v}_{x_{k}}^{2} d x d t \\
\leq \int_{0}^{T}\left\|\vec{v}^{N}-\vec{v}\right\|_{4, \Omega}^{2} \cdot\left\|\vec{v}_{x}\right\|_{4, \Omega}^{2} d t \\
\leq c(\Omega) \int_{0}^{T}\left\|\left(\vec{v}^{N}-\vec{v}\right)_{x}\right\|_{2, \Omega}^{2} \cdot\left\|\vec{v}_{x x}\right\|_{2, \Omega}^{2} d t \\
\leq c(\Omega) \sup _{[0, T]}\left(\|\vec{v}\|_{2, \Omega}^{(2)}\right)^{2} \cdot\left\|\left(\vec{v}^{N}-\vec{v}\right)_{x}\right\|_{2, Q_{T}}^{2}, \\
\left\|h_{k} \vec{H}_{x_{k}}\right\|_{2, Q_{T}}^{2}=\left\|\left(\vec{H}^{N}-\vec{H}\right)_{k} \cdot \vec{H}_{x_{k}}\right\|_{2, Q_{T}}^{2} \\
\leq c(\Omega) \sup _{[0, T]}\left(\|\vec{H}\|_{2, \Omega}^{(2)}\right)^{2}\left\|\left(\vec{H}^{N}-\vec{H}\right)_{x}\right\|_{2, Q_{T}}^{2} .
\end{aligned}
$$

For the difference of two generalized solutions of problems [9] the following inequality holds:

$$
\begin{aligned}
\chi(t) \leq & \int_{0}^{t}\left(\sqrt{\rho}\left\|\left(\vec{f}^{N}-\vec{f}\right) g(x, \tau)\right\|+\frac{1}{\sqrt{\sigma}}\left\|\left(\vec{r}^{N}-\vec{r}\right) \xi(x, \tau)\right\|\right) \exp \left\{c_{0} \int_{\tau}^{t} \Phi^{\prime \prime 2}(s) d s\right\} d \tau \\
= & \int_{0}^{t}\left[\sqrt{\rho}\left(\int_{\Omega}\left(\vec{f}^{N}-\vec{f}\right)^{2}|g|^{2} d x\right)^{\frac{1}{2}}\right. \\
& \left.+\frac{1}{\sqrt{\sigma}}\left(\int_{\Omega}\left(\vec{r}^{N}-\vec{r}\right)^{2}|\xi|^{2} d x\right)^{\frac{1}{2}}\right] d \tau \exp \left\{c_{0} \int_{\tau}^{t} \Phi^{\prime \prime 2}(s) d s\right\} d \tau
\end{aligned}
$$




$$
\begin{aligned}
\leq & \exp \left\{c_{0} \int_{0}^{T} \Phi^{\prime \prime 2}(t) d t\right\} \int_{0}^{t}\left[\sqrt{\rho}\left\|\vec{f}^{N}-\vec{f}\right\| \sup _{\Omega}|g(x, \tau)|\right. \\
& \left.+\frac{1}{\sqrt{\sigma}}\left\|\vec{r}^{N}-\vec{r}\right\| \sup _{\Omega}|\xi(x, \tau)|\right] d \tau \\
\leq & \exp \left\{c_{0} \int_{0}^{T} \Phi^{\prime \prime 2}(t) d t\right\}\left(\sqrt{\rho}\left\|\vec{f}^{N}-\vec{f}\right\| \int_{0}^{T} \sup _{\Omega}|g(x, \tau)| d \tau\right. \\
+ & \left.\frac{1}{\sqrt{\sigma}}\left\|\vec{r}^{N}-\vec{r}\right\| \int_{0}^{T} \sup |\xi(x, \tau)| d \tau\right), \\
\int_{0}^{t} \Phi^{2}(s) d s \leq & 2 c_{0} \int_{0}^{t} \chi^{2}(s) \Phi^{\prime \prime 2}(s) d s \\
& +\int_{0}^{t} \chi(s)\left(\sqrt{\rho}\|\vec{f}\|+\frac{1}{\sqrt{\sigma}}\|\operatorname{rot} \vec{j}\|\right) d s,
\end{aligned}
$$

where

$$
\begin{aligned}
& \chi^{2}(t)=\rho\|\vec{W}\|^{2}+\mu\|\vec{h}\|^{2}, \quad \Phi^{2}(t)=\rho v\left\|\vec{W}_{x}\right\|^{2}+\frac{1}{\sigma}\|\operatorname{rot} \vec{h}\|^{2}, \\
& \Phi^{\prime \prime 2}(t)=\rho v\left\|\vec{v}_{x}\right\|^{2}+\frac{1}{\sigma}\|\operatorname{rot} \vec{H}\|^{2}, \\
& \vec{f}=\left(\vec{f}^{N}(x)-\vec{f}(x)\right) g(x, t), \quad \operatorname{rot} \vec{j}=\left(\vec{r}^{N}(x)-\vec{r}(x)\right) \xi(x, t), \quad \chi(0)=0 .
\end{aligned}
$$

By squaring and integrating both sides of inequality (20) on $t$ from 0 to $T$, we have

$$
\begin{aligned}
\int_{0}^{T} \chi^{2}(t) d t= & \rho\|\vec{W}\|_{2, Q_{T}}^{2}+\mu\|\vec{h}\|_{2, Q_{T}}^{2} \\
\leq & 2 T \exp \left\{2 c_{0} \int_{0}^{T} \Phi^{\prime \prime 2}(t) d t\right\}\left(\rho\left\|\vec{f}^{N}-\vec{f}\right\|^{2}\left(\int_{0}^{T} \sup _{\Omega}|g(x, t)| d t\right)^{2}\right. \\
& \left.+\frac{1}{\sigma}\left\|\vec{r}^{N}-\vec{r}\right\|^{2}\left(\int_{0}^{T} \sup _{\Omega}|\xi(x, t)| d t\right)^{2}\right)
\end{aligned}
$$

then

$$
\begin{aligned}
\int_{0}^{T} \Phi^{2}(t) d t= & \rho v\left\|\left(\vec{v}^{N}-\vec{v}\right)_{x}\right\|_{2, Q_{T}}^{2}+\frac{1}{\sigma}\left\|\operatorname{rot} \vec{H}^{N}-\operatorname{rot} \vec{H}\right\|_{2, Q_{T}}^{2} \\
\leq & 2 c_{0} \int_{0}^{T} \chi^{2}(t) \Phi^{\prime \prime 2}(t) d t \\
& +\int_{0}^{T} \chi(t)\left(\sqrt{\rho}\left\|\left(\vec{f}^{N}-\vec{f}\right) g(x, t)\right\|+\frac{1}{\sqrt{\sigma}}\left\|\left(\vec{r}^{N}-\vec{r}\right) \xi(x, t)\right\|\right) d t \\
\leq & 4 T c_{0} \exp \left\{2 c_{0} \int_{0}^{T} \Phi^{\prime \prime 2}(t) d t\right\} \int_{0}^{T}\left(\rho\left\|\vec{f}^{N}-\vec{f}\right\|^{2}\left(\int_{0}^{t} \sup |g(x, \tau)| d \tau\right)^{2}\right. \\
& \left.+\frac{1}{\sigma}\left\|\vec{r}^{N}-\vec{r}\right\|^{2}\left(\int_{0}^{t} \sup _{\Omega}|\xi(x, \tau)| d \tau\right)^{2}\right) \Phi^{\prime 2}(t) d t \\
& +2 \sqrt{2 T} \exp \left\{c_{0} \int_{0}^{T} \Phi^{\prime \prime 2}(t) d t\right\}\left(\rho\left\|\vec{f}^{N}-\vec{f}\right\|^{2}\left(\int_{0}^{T} \sup _{\Omega}|g(x, t)| d t\right)^{2}\right. \\
& \left.+\frac{1}{\sigma}\left\|\vec{r}^{N}-\vec{r}\right\|^{2}\left(\int_{0}^{T} \sup _{\Omega}|\xi(x, t)| d t\right)^{2}\right)^{\frac{1}{2}}
\end{aligned}
$$




$$
\begin{aligned}
& \times\left(\int _ { 0 } ^ { T } \left(\rho\left\|\vec{f}^{N}-\vec{f}\right\|^{2}\left(\sup _{\Omega}|g(x, t)|\right)^{2}\right.\right. \\
& \left.\left.+\frac{1}{\sigma}\left\|\vec{r}^{N}-\vec{r}\right\|^{2}\left(\sup _{\Omega}|\xi(x, t)|\right)^{2}\right) d t\right)^{\frac{1}{2}} .
\end{aligned}
$$

Furthermore, (12), (22), (23), and (19) imply that

$$
\left\|\left(\vec{v}^{N}-\vec{v}\right)_{t}\right\|_{2, Q_{T}}^{2}+\left\|\left(\vec{H}^{N}-\vec{H}\right)_{t}\right\|_{2, Q_{T}}^{2} \rightarrow 0, \quad \text { as } N \rightarrow \infty .
$$

Let us consider in $Q_{T}$ the problem

$$
\begin{aligned}
& \vec{W}_{t}-v \Delta \vec{W}=-\frac{1}{\rho} \nabla P+\vec{F}_{1}, \quad \operatorname{div} \vec{W}=0, \\
& \left.\vec{W}\right|_{t=0}=\vec{v}_{2}(x),\left.\quad \vec{W}\right|_{\Gamma}=0, \\
& \vec{h}_{t}+\frac{1}{\sigma \mu} \operatorname{rot} \operatorname{rot} \vec{h}=\vec{G}_{1}, \quad \operatorname{div} \vec{h}=0, \\
& \left.h_{n}\right|_{S}=0,\left.\quad \operatorname{rot} \vec{h}\right|_{\Gamma}=0,\left.\quad \vec{h}\right|_{t=0}=\vec{h}_{2}(x),
\end{aligned}
$$

where

$$
\begin{aligned}
& \vec{W}=\left(\vec{v}^{N}-\vec{v}\right)_{t}, \quad \vec{h}=\left(\vec{H}^{N}-\vec{H}\right)_{t}, \\
& \vec{F}_{1}=\frac{\partial}{\partial t}\left[\left(\vec{f}^{N}-\vec{f}\right) g-v_{k}^{N} \vec{v}_{x_{k}}^{N}+v_{k} \vec{v}_{x_{k}}+\frac{\mu}{\rho}\left(H_{k}^{N} \vec{H}_{x_{k}}^{N}-H_{k} \vec{H}_{x_{k}}\right)\right], \\
& \vec{G}_{1}=\frac{\partial}{\partial t}\left[\frac{\xi(x, t)}{\sigma \mu}\left(\vec{r}^{N}-\vec{r}\right)+\left(\operatorname{rot}\left[\vec{v}^{N} \times \vec{H}^{N}\right]-\operatorname{rot}[\vec{v} \times \vec{H}]\right)\right], \\
& \vec{v}_{2}(x)=P\left(\vec{f}^{N}-\vec{f}\right) g(x, 0), \quad \vec{h}_{2}(x)=P\left[\vec{r}^{N}-\vec{r}\right] \frac{\xi(x, 0)}{\sigma \mu} .
\end{aligned}
$$

The problem (25)-(26), as well as the problem (27)-(28) with the conditions in the space $V_{2}\left(Q_{T}\right)$ is uniquely solvable $[4,9,13]$. We now take the dot product of the first equation of (25) with the function $\vec{W}(x, t)$ and of (27) with $\vec{h}(x, t)$, respectively, in $L_{2}\left(Q_{T}\right)$, then we obtain

$$
\begin{gathered}
\frac{1}{2}\|\vec{W}(x, T)\|_{2, \Omega}^{2}+v \int_{0}^{T}\left\|\vec{W}_{x}(x, t)\right\|_{2, \Omega}^{2} d t \\
=\int_{Q_{T}} \vec{F}_{1} \vec{W} d x d t+\frac{1}{2}\|\vec{W}(x, 0)\|_{2, \Omega}^{2}, \\
\frac{1}{2}\|\vec{h}(x, T)\|_{2, \Omega}^{2}+v \int_{0}^{T}\|\operatorname{rot} \vec{h}(x, t)\|_{2, \Omega}^{2} d t \\
=\int_{Q_{T}} \vec{G} \vec{W} d x d t+\frac{1}{2}\|\vec{h}(x, 0)\|_{2, \Omega}^{2} .
\end{gathered}
$$

Using the method of [4] we prove that $\left(\vec{v}^{N}-\vec{v}\right)_{t}$ and $\left(\vec{H}^{N}-\vec{H}\right)_{t}$ are the solutions of the problems (23)-(24) and (25)-(26) from $V_{2}\left(Q_{T}\right)$. Estimating the right-hand sides of (29) and (30), and by (12) and (24), we have

$$
\left\|\left(\vec{v}^{N}-\vec{v}\right)_{t}(x, T)\right\|_{2, Q_{T}}^{2}+\left\|\left(\vec{H}^{N}-\vec{H}\right)_{t}(x, T)\right\|_{2, Q_{T}}^{2} \rightarrow 0, \quad \text { as } N \rightarrow \infty .
$$

Thus we have proved that the operators $T_{g}$ and $S_{\xi}$ are continuous. 
Now we show the complete continuity of the operators $T_{g}$ and $S_{\xi}$. Let us consider the following problem:

$$
\begin{aligned}
& \vec{W}_{t}-v \Delta \vec{W}=-\frac{1}{\rho} \nabla P+\vec{F}_{1}, \quad \operatorname{div} \vec{W}=0, \\
& \vec{h}_{t}+\frac{1}{\sigma \mu} \operatorname{rot} \operatorname{rot} \vec{h}=\vec{G}_{1}, \quad \operatorname{div} \vec{h}=0, \\
& \left.h_{n}\right|_{\Gamma}=\left.H_{n}\right|_{\Gamma}=0,\left.\quad \operatorname{rot} \vec{h}\right|_{\Gamma}=0,\left.\quad \vec{W}\right|_{\Gamma}=0, \\
& \left.\vec{W}\right|_{t=0}=\vec{v}_{2}(x),\left.\quad \vec{h}\right|_{t=0}=\vec{h}_{2}(x) .
\end{aligned}
$$

Here

$$
\begin{aligned}
& \vec{W}=\vec{v}_{t}, \quad \vec{h}=\vec{H}_{t}, \quad \vec{F}_{1}=\frac{\partial}{\partial t}\left[\vec{f}(x) g(x, t)-v_{k} \vec{v}_{x_{k}}+\frac{\mu}{\rho} H_{k} \vec{H}_{x_{k}}\right], \\
& \vec{G}_{1}=\frac{\partial}{\partial t}\left[\frac{\xi(x, t)}{\sigma \mu} \vec{r}(x)+\operatorname{rot}[\vec{v} \times \vec{H}]\right], \\
& \vec{v}_{2}(x)=P\left(v \Delta \vec{v}_{0}+\vec{f}(x) g(x, 0)-v_{0 k} \vec{v}_{0 x_{k}}+\frac{\mu}{\rho} H_{0 k} \vec{H}_{0 x_{k}}\right), \\
& \vec{h}_{2}(x)=P\left[\frac{1}{\sigma \mu} \operatorname{rot} \operatorname{rot} \vec{H}_{0}+\frac{\xi(x, 0)}{\sigma \mu} \vec{r}(x)+\operatorname{rot}\left[\vec{v}_{0} \times \vec{H}_{0}\right]\right] .
\end{aligned}
$$

By the differential conditions of the problem (32)-(34) we fix an arbitrary number $\varepsilon$. Since the functions $\left\|\vec{W}_{x}(x, t)\right\|_{2, \Omega}$ and $\|\operatorname{rot} \vec{h}(x, t)\|_{2, \Omega}$ are continuous on $[\varepsilon, T]$, we could choose $t_{*} \in[\varepsilon, T]$ such that the following equalities hold:

$$
\begin{aligned}
& \int_{\varepsilon}^{T}\left\|\vec{W}_{x}(x, t)\right\|_{2, \Omega}^{2} d t=(T-\varepsilon)\left\|\vec{W}_{x}\left(x, t_{*}\right)\right\|_{2, \Omega}^{2} \\
& \int_{\varepsilon}^{T}\|\operatorname{rot} \vec{h}(x, t)\|_{2, \Omega}^{2} d t=(T-\varepsilon)\left\|\operatorname{rot} \vec{h}\left(x, t_{*}\right)\right\|_{2, \Omega}^{2}
\end{aligned}
$$

Equations (32) and (33) imply that

$$
\begin{aligned}
& \int_{t_{*}}^{T} \int_{\Omega}\left|\vec{W}_{t}-v P \Delta \vec{W}\right|^{2} d x d t=\int_{t_{*}}^{T} \int_{\Omega}\left|P \vec{F}_{1}\right|^{2} d x d t, \\
& \int_{t_{*}}^{T} \int_{\Omega}\left|\vec{h}_{t}+\frac{1}{\sigma \mu} P \operatorname{rot} \operatorname{rot} \vec{h}\right|^{2} d x d t=\int_{t_{*}}^{T} \int_{\Omega}\left|P \vec{G}_{1}\right|^{2} d x d t .
\end{aligned}
$$

Integrating by parts (36), we obtain

$$
\begin{aligned}
& v\left\|\vec{W}_{x}(x, T)\right\|_{2, \Omega}^{2}+\int_{t_{*}}^{T} \int_{\Omega}\left(\left|\vec{W}_{t}\right|^{2}+|v P \Delta \vec{W}|^{2}\right) d x d t \\
& \quad=\int_{t_{*}}^{T} \int_{\Omega}\left|P \vec{F}_{1}\right|^{2} d x d t+v\left\|\vec{W}_{x}\left(x, t_{*}\right)\right\|_{2, \Omega^{\prime}}^{2} \\
& \frac{1}{\sigma \mu}\|\operatorname{rot} \vec{h}(x, T)\|_{2, \Omega}^{2}+\int_{t_{*}}^{T} \int_{\Omega}\left(\left|\vec{h}_{t}\right|^{2}+\left|\frac{1}{\sigma \mu} P \operatorname{rot} \operatorname{rot} \vec{h}\right|^{2}\right) d x d t \\
& \quad=\int_{t_{*}}^{T} \int_{\Omega}\left|P \vec{G}_{1}\right|^{2} d x d t+\frac{1}{\sigma \mu}\left\|\operatorname{rot} \vec{h}\left(x, t_{*}\right)\right\|_{2, \Omega^{\prime}}^{2}
\end{aligned}
$$


By equality (35) and equality (37), we obtain

$$
\begin{aligned}
& v\left\|\vec{W}_{x}(x, T)\right\|_{2, \Omega}^{2} \leq \int_{t_{*}}^{T} \int_{\Omega}\left|P \vec{F}_{1}\right|^{2} d x d t+\frac{v}{T-\varepsilon}\left\|\vec{W}_{x}(x, t)\right\|_{2, Q_{T}}^{2}, \\
& \frac{1}{\sigma \mu}\|\operatorname{rot} \vec{h}(x, T)\|_{2, \Omega}^{2} \leq \int_{t_{*}}^{T} \int_{\Omega}\left|P \vec{G}_{1}\right|^{2} d x d t+\frac{1}{\sigma \mu(T-\varepsilon)}\|\operatorname{rot} \vec{h}(x, t)\|_{2, Q_{T}}^{2} .
\end{aligned}
$$

By the well-known theory in $[4,13]$, the following inequalities hold:

$$
\begin{aligned}
& v\left\|\vec{W}_{x}\right\|_{2, Q_{T}}^{2} \leq\left\|\vec{v}_{2}(x)\right\|_{2, \Omega}^{2}+\frac{3}{2}\left(\int_{0}^{T}\left\|\vec{F}_{1}(x, t)\right\|_{2, \Omega} d t\right)^{2}, \\
& \frac{1}{\sigma \mu}\|\operatorname{rot} \vec{h}\|_{2, Q_{T}}^{2} \leq\left\|\vec{h}_{2}(x)\right\|_{2, \Omega}^{2}+\frac{3}{2}\left(\int_{0}^{T}\left\|\vec{G}_{1}(x, t)\right\|_{2, \Omega} d t\right)^{2}
\end{aligned}
$$

for the solutions of problem (32)-(34).

Since $\Omega \subset R^{2}, g, g_{t} \in C\left(\bar{Q}_{T}\right), \xi, \xi_{t} \in C\left(\bar{Q}_{T}\right)$, and $\vec{f}(x)$, $\operatorname{rot} \vec{j}(x) \in L_{2}(\Omega)$, the following inequalities hold:

$$
\begin{aligned}
& \left\|\vec{f}(x) g(x, t)-v_{k} \vec{v}_{x_{k}}+\frac{\mu}{\rho} H_{k} \vec{H}_{x_{k}}\right\|_{2, \Omega} \\
& \leq\|\vec{f}(x) g(x, t)\|_{2, \Omega}+c(\Omega) \sup _{[0, T]}\|\vec{v}(x, t)\|_{2, \Omega}^{(2)} \cdot\left\|\vec{v}_{x}(x, t)\right\|_{2, \Omega} \\
& +c(\Omega) \sup _{[0, T]}\|\vec{H}(x, t)\|_{2, \Omega}^{(2)} \cdot\|\operatorname{rot} \vec{H}(x, t)\|_{2, \Omega}, \\
& \left\|\frac{\xi(x, t)}{\sigma \mu} \vec{r}(x)+\operatorname{rot}[\vec{v} \times \vec{H}]\right\|_{2, \Omega} \\
& \leq\left\|\frac{\xi(x, t)}{\sigma \mu} \vec{r}(x)\right\|_{2, \Omega}+c(\Omega) \sup _{[0, T]}\left(\|\vec{v}(x, t)\|_{2, \Omega}^{(2)}\right)^{2} \cdot\|\operatorname{rot} \vec{H}(x, t)\|_{2, \Omega}^{2} \\
& +c(\Omega) \sup _{[0, T]}\left(\|\vec{H}(x, t)\|_{2, \Omega}^{(2)}\right)^{2} \cdot\left\|\vec{v}_{x}(x, t)\right\|_{2, \Omega}^{2}, \\
& \left\|\vec{F}_{1}(x, t)\right\|_{2, \Omega} \leq\left\|\vec{f}(x) g_{t}(x, t)\right\|_{2, \Omega}+c_{1}(\Omega) \sup _{[0, T]}\|\vec{v}(x, t)\|_{2, \Omega}^{(2)} \cdot\left\|\vec{v}_{t x}(x, t)\right\|_{2, \Omega} \\
& +c_{1}(\Omega) \sup _{[0, T]}\|\vec{H}(x, t)\|_{2, \Omega}^{(2)} \cdot\left\|\operatorname{rot} \vec{H}_{t}(x, t)\right\|_{2, \Omega} \\
& \left\|\vec{G}_{1}(x, t)\right\|_{2, \Omega} \leq\left\|\frac{\xi_{t}(x, t)}{\sigma \mu} \vec{r}(x)\right\|_{2, \Omega}+c_{1}(\Omega) \sup _{[0, T]}\left(\|\vec{v}(x, t)\|_{2, \Omega}^{(2)}\right)^{2} \cdot\left\|\operatorname{rot} \vec{H}_{t}(x, t)\right\|_{2, \Omega}^{2} \\
& +c_{1}(\Omega) \sup _{[0, T]}\left(\|\vec{H}(x, t)\|_{2, \Omega}^{(2)}\right)^{2} \cdot\left\|\vec{v}_{t x}(x, t)\right\|_{2, \Omega}^{2} .
\end{aligned}
$$

By inequalities (39) and (40), we obtain

$$
\begin{aligned}
v\left\|\vec{W}_{x}(x, T)\right\|_{2, \Omega}^{2} \leq & \left(1+\frac{3}{2} T^{2}\right)\left\|\vec{F}_{1}\right\|_{2, Q_{T}}^{2}+\frac{1}{T-\varepsilon}\left[2\left\|v \Delta \vec{v}_{0}-v_{0 k} \vec{v}_{0 x_{k}}+\frac{\mu}{\rho} H_{0 k} \vec{H}_{0 x_{k}}\right\|_{2, \Omega}^{2}\right. \\
& +6\|\vec{f}(x) g(x, 0)\|_{2, \Omega}^{2}+6\left(c(\Omega) \sup _{[0, T]}\|\vec{v}\|_{2, \Omega}^{(2)}\right)^{2}\left\|\vec{v}_{0 x}\right\|_{2, \Omega}^{2}
\end{aligned}
$$




$$
\begin{aligned}
+ & \left.6\left(c(\Omega) \sup _{[0, T]}\|\vec{H}\|_{2, \Omega}^{(2)}\right)^{2}\left\|\operatorname{rot} \vec{H}_{0}\right\|_{2, \Omega}^{2}\right] \\
\frac{1}{\sigma \mu}\|\operatorname{rot} \vec{h}(x, T)\|_{2, \Omega}^{2} \leq & \left(1+\frac{3}{2} T^{2}\right)\left\|\vec{G}_{1}\right\|_{2, Q_{T}}^{2} \\
& +\frac{1}{T-\varepsilon}\left[2\left\|\frac{1}{\sigma \mu} \operatorname{rot} \operatorname{rot} \vec{H}_{0}+\operatorname{rot}\left[\vec{v}_{0} \times \vec{H}_{0}\right]\right\|_{2, \Omega}^{2}\right. \\
& +6\left\|\frac{\xi(x, 0)}{\sigma \mu} \vec{r}\right\|_{2, \Omega}^{2}+6\left(c(\Omega) \sup _{[0, T]}\|\vec{H}\|_{2, \Omega}^{(2)}\right)^{2}\left\|\vec{v}_{0 x}\right\|_{2, \Omega}^{2} \\
& \left.+6\left(c(\Omega) \sup _{[0, T]}\|\vec{v}\|_{2, \Omega}^{(2)}\right)^{2}\left\|\operatorname{rot} \vec{H}_{0}\right\|_{2, \Omega}^{2}\right] .
\end{aligned}
$$

Moreover, the equality (41) implies that

$$
\begin{aligned}
\left\|\vec{F}_{1}(x, t)\right\|_{2, Q_{T}}^{2} \leq & 3\left\|\vec{f}(x) g_{t}(x, t)\right\|_{2, Q_{T}}^{2} \\
& +3\left(c_{1}(\Omega) \sup _{[0, T]}\|\vec{v}\|_{2, \Omega}^{2}\right)^{2} \cdot\left\|\vec{v}_{t x}(x, t)\right\|_{2, Q_{T}}^{2} \\
& +3\left(c_{1}(\Omega) \sup _{[0, T]}\|\vec{H}(x, t)\|_{2, \Omega}^{(2)}\right)^{2} \cdot\left\|\operatorname{rot} \vec{H}_{t}(x, t)\right\|_{2, Q_{T}}^{2}, \\
\left\|\vec{G}_{1}(x, t)\right\|_{2, Q_{T}}^{2} \leq & \left\|\frac{\xi_{t}(x, t)}{\sigma \mu} \vec{r}(x)\right\|_{2, Q_{T}}^{2} \\
& +3\left(c_{1}(\Omega) \sup _{[0, T]}\left(\|\vec{v}(x, t)\|_{2, \Omega}^{(2)}\right)^{2}\right)^{2} \cdot\left\|\operatorname{rot} \vec{H}_{t}(x, t)\right\|_{2, Q_{T}}^{2} \\
& +3\left(c_{1}(\Omega) \sup _{[0, T]}\left(\|\vec{H}(x, t)\|_{2, \Omega}^{(2)}\right)^{2}\right) \cdot\left\|\vec{v}_{t x}(x, t)\right\|_{2, Q_{T}}^{2} .
\end{aligned}
$$

Here $\left\|\vec{F}_{1}\right\|_{2, Q_{T}}^{2}$ and $\left\|\vec{G}_{1}\right\|_{2, Q_{T}}^{2}$ are bounded, since one can estimate $\vec{v}_{t}(x, t), \vec{v}_{t x}(x, t), \vec{H}_{t}(x, t)$ and $\operatorname{rot} \vec{H}_{t}$ in $L_{2}\left(Q_{T}\right)$ via the data of the problem and $\|\vec{f}\|_{2, \Omega},\|\vec{r}\|_{2, \Omega}$. By combining (40) and (41), we have

$$
\left\|\vec{v}_{t x}(x, T)\right\|_{2, \Omega}^{2}+\left\|\operatorname{rot} \vec{H}_{t}(x, T)\right\|_{2, \Omega}^{2} \leq M_{0} .
$$

Thus, the operators $T_{g}$ and $S_{\xi}$ map any bounded set $D$ from $L_{2}(\Omega)$, which is the domain of definition of $T_{g}$ and $S_{\xi}$ into a bounded set $\tilde{D}$ from $W_{2}^{1}(\Omega)$. Then the set $\tilde{D}$ is compact in $L_{2}(\Omega)$ by the Rellich theorem. Thus the operators $T_{g}$ and $S_{\xi}$ are continuous and map every bounded set into a compact set. Consequently, the operators $T_{g}$ and $S_{\xi}$ are completely continuous. The operators $A$ and $B$ are also completely continuous as a composition of linear bounded and completely continuous operators.

Theorem 4 If $g, g_{t} \in C\left(\bar{Q}_{T}\right), \xi, \xi_{t} \in C\left(\bar{Q}_{T}\right),|g(x, t)| \geq g_{T}>0,|\xi(x, t)| \geq \xi_{T}>0$ as $x \in \Omega$, $\vec{U}(x) \in W_{2}^{2}(\Omega) \cap J_{1}(\Omega), \vec{H}_{0}(x) \in \hat{J}(\Omega), \vec{\Psi}(x) \in \hat{J}(\Omega), \vec{v}_{0}(x) \in W_{2}^{2}(\Omega) \cap \stackrel{0}{J}_{1}(\Omega), \nabla \pi(x) \in G(\Omega)$. Let the inequalities

$$
\frac{1}{\sigma}>\mu c_{1}\left(\|\vec{U}\|_{4, \Omega}+\|\vec{\Psi}\|_{4, \Omega}\right), \quad v>c_{1}\left(\|\vec{U}\|_{4, \Omega}+\frac{\mu}{\rho}\|\vec{\Psi}\|_{4, \Omega}\right)
$$

hold. Then the problem (1)-(6) is solvable if and only if (11) is solvable in $L_{2}(\Omega)$. 
Proof We suppose that the operator equations (11) are solvable. We introduce the notation $\vec{f}_{1}(x)$ and $\vec{r}_{1}=\operatorname{rot} \vec{j}_{1}(x)$. Then by [9-15], we can find $\vec{v}_{1}, \nabla p_{1}$, and $\vec{H}_{1}$ in the necessary classes of functions, satisfying (1)-(5) with vectors $\vec{f}_{1}(x) g(x, t)$ and $\xi(x, t) \operatorname{rot} \vec{j}_{1}(x)$. We show that these functions satisfy the overdetermination condition (6), also. Let $\vec{v}(x, T)=\vec{U}_{1}(x)$, $\vec{H}(x, T)=\vec{\Psi}_{1}(x), \nabla p(x, T)=\nabla \pi(x)$, then for the function $\vec{w}=\vec{U}-\vec{U}_{1}, \vec{z}=\vec{\Psi}-\vec{\Psi}_{1}$, we have the following problem:

$$
\begin{aligned}
& -v \Delta \vec{w}+w_{k} \vec{U}_{x_{k}}+U_{1 k} \vec{w}_{x_{k}}-\frac{\mu}{\rho} z_{k} \vec{\Psi}_{x_{k}}-\frac{\mu}{\rho} \Psi_{1 k} \vec{z}_{x_{k}}=-\nabla q, \\
& \frac{1}{\sigma \mu} \operatorname{rot} \operatorname{rot} \vec{z}+w_{k} \vec{\Psi}_{x_{k}}+U_{1 k} \vec{z}_{x_{k}}-z_{k} \vec{U}_{x_{k}}-\Psi_{1 k} \vec{w}_{x_{k}}=0, \\
& \operatorname{div} \vec{w}=0, \quad \operatorname{div} \mu \vec{z}=0, \\
& \left.\vec{w}\right|_{\Gamma}=0,\left.\quad z_{n}\right|_{\Gamma}=0, \quad \frac{\partial z_{2}}{\partial x_{1}}-\left.\frac{\partial z_{1}}{\partial x_{2}}\right|_{\Gamma}=0 .
\end{aligned}
$$

Now we take the dot product of (46) with the function $\vec{w}$ and of (47) with $\vec{z}$, respectively, in $L_{2}(\Omega)$,

$$
\begin{gathered}
\nu \rho\left\|\vec{w}_{x}\right\|^{2}+\rho \int_{\Omega} w_{k} \vec{U}_{x_{k}} \vec{w} d x+\rho \int_{\Omega} w_{x_{k}} U_{1 k} \vec{w} d x \\
-\mu \int_{\Omega} z_{k} \vec{\Psi}_{x_{k}} \vec{w} d x-\mu \int_{\Omega} \Psi_{1 k} \vec{z}_{x_{k}} \vec{w} d x=0, \\
\frac{1}{\sigma}\|\operatorname{rot} \vec{z}\|^{2}-\mu \int_{\Omega} w_{k} \vec{\Psi}_{x_{k}} \vec{z} d x+\mu \int_{\Omega} U_{1 k} \vec{z}_{x_{k}} \vec{z} d x \\
-\mu \int_{\Omega} z_{k} \vec{U}_{x_{k}} \vec{z} d x-\mu \int_{\Omega} \vec{w}_{x_{k}} \Psi_{1 k} \vec{z} d x=0 .
\end{gathered}
$$

We transform the previous equalities into the following form:

$$
\begin{aligned}
& \nu \rho\left\|\vec{w}_{x}\right\|^{2}-\rho \int_{\Omega} w_{k} \vec{U} \vec{w}_{x_{k}} d x+\mu \int_{\Omega} z_{k} \vec{\Psi} \vec{w}_{x_{k}} d x-\mu \int_{\Omega} \Psi_{1 k} \vec{z}_{x_{k}} \vec{w} d x=0, \\
& \frac{1}{\sigma}\|\operatorname{rot} \vec{z}\|^{2}-\mu \int_{\Omega} w_{k} \vec{\Psi} \vec{z}_{x_{k}} d x+\mu \int_{\Omega} \vec{U} z_{k} \vec{z}_{x_{k}} d x+\mu \int_{\Omega} \vec{w} \Psi_{1 k} \vec{z}_{x_{k}} d x=0 .
\end{aligned}
$$

Now we add (50) and (51),

$$
\begin{aligned}
& v \rho\left\|\vec{w}_{x}\right\|^{2}+\frac{1}{\sigma}\|\operatorname{rot} \vec{z}\|^{2} \\
& =\rho \int_{\Omega} w_{k} \vec{U} \vec{w}_{x_{k}} d x-\mu \int_{\Omega} z_{k} \vec{\Psi} \vec{w}_{x_{k}} d x \\
& \quad+\mu \int_{\Omega} w_{k} \vec{\Psi} \vec{z}_{x_{k}} d x-\mu \int_{\Omega} \vec{U} z_{k} \vec{z}_{x_{k}} d x
\end{aligned}
$$

the right-hand side of previous equality implies that

$$
\begin{aligned}
& v \rho\left\|\vec{w}_{x}\right\|^{2}+\frac{1}{\sigma}\|\operatorname{rot} \vec{z}\|^{2} \\
& \quad \leq \rho c_{1}\|\vec{U}\|_{4, \Omega}\left\|\vec{w}_{x}\right\|_{2, \Omega}^{2}+\mu\|\vec{w}\|_{4, \Omega}\|\vec{\Psi}\|_{4, \Omega}\left\|\vec{z}_{x}\right\|_{2, \Omega}
\end{aligned}
$$




$$
\begin{aligned}
& +\mu\|\vec{z}\|_{4, \Omega}\|\vec{\Psi}\|_{4, \Omega}\left\|\vec{w}_{x}\right\|_{2, \Omega}+\mu c_{1}\|\vec{U}\|_{4, \Omega}\left\|\vec{z}_{x}\right\|_{2, \Omega}^{2} \\
\leq & \rho c_{1}\|\vec{U}\|_{4, \Omega}\left\|\vec{w}_{x}\right\|_{2, \Omega}^{2}+\mu c_{1}\|\vec{U}\|_{4, \Omega}\left\|\vec{z}_{x}\right\|_{2, \Omega}^{2} \\
& +\mu c_{1}\left\|\vec{w}_{x}\right\|_{2, \Omega}\|\vec{\Psi}\|_{4, \Omega}\left\|\vec{z}_{x}\right\|_{2, \Omega}+\mu c_{1}\left\|\vec{z}_{x}\right\|_{2, \Omega}\|\vec{\Psi}\|_{4, \Omega}\left\|\vec{w}_{x}\right\|_{2, \Omega} \\
\leq & \rho c_{1}\|\vec{U}\|_{4, \Omega}\left\|\vec{w}_{x}\right\|_{2, \Omega}^{2}+\mu c_{1}\|\vec{U}\|_{4, \Omega}\left\|\vec{z}_{x}\right\|_{2, \Omega}^{2} \\
& +\mu c_{1}\|\vec{\Psi}\|_{4, \Omega}\left(\left\|\vec{w}_{x}\right\|_{2, \Omega}^{2}+\left\|\vec{z}_{x}\right\|_{2, \Omega}^{2}\right) .
\end{aligned}
$$

Hence

$$
\begin{aligned}
& \left(v \rho-c_{1}\left(\rho\|\vec{U}\|_{4, \Omega}+\mu\|\vec{\Psi}\|_{4, \Omega}\right)\right)\left\|\vec{w}_{x}\right\|^{2} \\
& \quad+\left(\frac{1}{\sigma}-\mu c_{1}\left(\|\vec{U}\|_{4, \Omega}+\|\vec{\Psi}\|_{4, \Omega}\right)\right)\|\operatorname{rot} \vec{z}\|^{2} \leq 0 .
\end{aligned}
$$

By conditions (45), from inequality (52) we obtain $\vec{U}=\vec{U}_{1}, \vec{\Psi}=\vec{\Psi}_{1}$, then $\nabla \pi(x)=\nabla \pi_{1}(x)$. Thus, $\vec{v}_{1}, \nabla p_{1}, \vec{H}_{1}, \vec{f}_{1}(x)$, and $\vec{r}_{1}=\operatorname{rot} \vec{j}_{1}(x)$ satisfy all conditions (1)-(6). Consequently, the problem (1)-(6) is solvable.

Necessity. Assume that the problem (1)-(6) is solvable. Let us denote this solution by $\{\vec{v}, \nabla p, \vec{H}, \operatorname{rot} \vec{j}\}$. Hence, we obtain the operator equations (11). Furthermore, we see that $\vec{f}(x)$ and $\vec{r}=\operatorname{rot} \vec{j}(x)$ are solutions of this equation.

Theorem 5 Let the condition of Theorem 4 hold. Let the following inequality hold:

$$
M_{1}+\|\vec{\aleph}\|_{2, \Omega}+\|\vec{\lambda}\|_{2, \Omega}<1,
$$

where

$$
\begin{aligned}
&\|\Phi\|_{2, Q_{T}}^{2}=v\left\|\vec{v}_{x}\right\|_{2, Q_{T}}^{2}+\frac{1}{\sigma}\|\operatorname{rot} \vec{H}\|_{2, Q_{T}}^{2}, \quad \beta=\frac{2-\varepsilon}{2 d^{2}}, \quad \varepsilon \in(0,2], \\
& \vec{\aleph}=\frac{1}{g(x, T)}\left[-v \Delta \vec{U}+U_{k} \vec{U}_{x_{k}}-\frac{\mu}{\rho} \Psi_{k} \vec{\Psi}_{x_{k}}+\frac{1}{\rho} \nabla\left(\pi+\frac{\mu \vec{\Psi}^{2}}{2}\right)\right], \\
& \vec{\lambda}=\frac{\sigma \mu}{\xi(x, T)}\left[\frac{1}{\sigma \mu} \operatorname{rot} \operatorname{rot} \vec{h}-\operatorname{rot}(\vec{U} \times \vec{\Psi})\right], \\
& M_{1}=\exp \left\{\frac{c^{2}}{2 d}\|\Phi\|_{2, Q_{T}}^{2}\right\}\left(\frac{1}{\inf _{\Omega}|g(x, T)|}+\frac{1}{\inf \Omega}|\xi(x, T)|\right. \\
& \times\left[\left(\left\|v \Delta \vec{v}_{0}-v_{0 k} \vec{v}_{0 x_{k}}+\frac{\mu}{\rho} H_{0 k} \vec{H}_{0 x_{k}}\right\|_{2, \Omega}\right.\right. \\
&+\sup _{\Omega}\left(|g(x, 0)|+\frac{1}{\sigma \mu}|\xi(x, 0)|\right) \\
&\left.+\left\|\frac{1}{\sigma \mu} \operatorname{rot} \operatorname{rot} \vec{H}_{0}+\operatorname{rot}\left[\vec{v}_{0} \times \vec{H}_{0}\right]\right\|\right) \exp \{-\beta T\} \\
&\left.+\int_{0}^{T} \exp \{-\beta(T-t)\} \sup _{\Omega}\left(\left|g_{t}(x, t)\right|+\frac{1}{\sqrt{\sigma}}\left|\xi_{t}(x, t)\right|\right) d t\right] .
\end{aligned}
$$

Then there exists a solution of the inverse problem (1)-(6). 
Proof It is known that for the direct problem (1)-(5), one can obtain the following inequality:

$$
\begin{aligned}
\chi^{2}(t) & +\int_{0}^{t} \Phi^{2}(\tau) d \tau \\
\leq & \rho\left\|\vec{v}_{0}\right\|_{2, \Omega}^{2}+\mu\left\|\vec{H}_{0}\right\|_{2, \Omega}^{2} \\
& +\frac{3}{2}\left\{\rho\left(\int_{0}^{T} \sup _{\Omega}|g(x, t)| d t\right)^{2}+\frac{1}{\sigma^{2} \mu}\left(\int_{0}^{T} \sup _{\Omega}|\xi(x, t)| d t\right)^{2}\right\} .
\end{aligned}
$$

We return to the problem (32)-(34). We rewrite (32), (33). By differentiating (1) and (2) with respect to $t$, we have

$$
\begin{aligned}
& \vec{v}_{t t}+\left(v_{k t} \vec{v}_{x_{k}}+v_{k} \vec{v}_{x_{k} t}\right)-\frac{\mu}{\rho}\left(H_{k t} \vec{H}_{x_{k}}+H_{k} \vec{H}_{x_{k} t}\right)-v \Delta \vec{v}_{t} \\
& \quad=-\frac{1}{\rho} \operatorname{grad}\left(p+\frac{\mu \vec{H}^{2}}{2}\right)_{t}+\vec{f} g_{t}, \\
& \vec{H}_{t t}+\frac{1}{\sigma \mu} \operatorname{rot} \operatorname{rot} \vec{H}_{t}-(\operatorname{rot}[\vec{v} \times \vec{H}])_{t}=\frac{1}{\sigma \mu} \xi_{t} \operatorname{rot} \vec{j}, \\
& (\operatorname{rot}[\vec{v} \times \vec{H}])_{t}=H_{k t} \vec{v}_{x_{k}}+H_{k} \vec{v}_{x_{k} t}-v_{k t} \vec{H}_{x_{k}}-v_{k} \vec{H}_{x_{k} t} .
\end{aligned}
$$

Taking the dot product of (55) and (56) with $\rho \vec{v}_{t}$ and $\mu \vec{H}_{t}$ in $L_{2}(\Omega)$, respectively, we obtain

$$
\begin{aligned}
& \frac{\rho}{2} \frac{d}{d t}\left\|\vec{v}_{t}\right\|^{2}+v\left\|\vec{v}_{t x}\right\|^{2}+\rho \int_{\Omega} v_{k t} \vec{v}_{x_{k}} \vec{v}_{t} d x \\
& \quad-\mu \int_{\Omega}\left(H_{k t} \vec{H}_{x_{k}}+H_{k} \vec{H}_{x_{k} t}\right) \vec{v}_{t} d x=\rho \int_{\Omega} \vec{f}_{g_{t}} \cdot \vec{v}_{t} d x, \\
& \frac{\mu}{2} \frac{d}{d t}\left\|\vec{H}_{t}\right\|^{2}+\frac{1}{\sigma}\left\|\operatorname{rot} \vec{H}_{t}\right\|^{2}-\mu \int_{\Omega} \vec{H}_{t} \cdot(\operatorname{rot}[\vec{v} \times \vec{H}])_{t} d x=\frac{1}{\sigma} \int_{\Omega} \vec{H}_{t} \cdot \xi_{t} \operatorname{rot} \vec{j} d x .
\end{aligned}
$$

Here

$$
\begin{aligned}
& \int_{\Omega} \vec{H}_{t} \cdot(\operatorname{rot}[\vec{v} \times \vec{H}])_{t} d x \\
& \quad=\int_{\Omega} \vec{H}_{t}\left(H_{k t} \vec{v}_{x_{k}}+H_{k} \vec{v}_{x_{k} t}-v_{k t} \vec{H}_{x_{k}}-v_{k} \vec{H}_{x_{k} t}\right) d x \\
& \quad=\int_{\Omega}\left(H_{k t} \vec{v}_{x_{k}} \vec{H}_{t}-H_{k} \vec{v}_{t} \vec{H}_{x_{k} t}-\vec{H}_{x_{k}} v_{k t} \vec{H}_{t}\right) d x .
\end{aligned}
$$

By combining (57) and (58), we obtain

$$
\frac{1}{2} \frac{d}{d t} \omega^{2}(t)+F^{2}(t)=I(t)+\rho \int_{\Omega} \vec{f} g_{t} \cdot \vec{v}_{t} d x+\frac{1}{\sigma} \int_{\Omega} \vec{H}_{t} \cdot \xi_{t} \operatorname{rot} \vec{j} d x
$$

Here we introduced the following notation:

$$
\begin{array}{ll}
\chi^{2}(t)=\rho\|\vec{v}\|^{2}+\mu\|\vec{H}\|^{2}, & \Phi^{2}(t)=\rho v\left\|\vec{v}_{x}\right\|^{2}+\frac{1}{\sigma}\|\operatorname{rot} \vec{H}\|^{2}, \\
\omega^{2}(t)=\rho\left\|\vec{v}_{t}\right\|^{2}+\mu\left\|\vec{H}_{t}\right\|^{2}, & F^{2}(t)=\rho v\left\|\vec{v}_{t x}\right\|^{2}+\frac{1}{\sigma}\left\|\operatorname{rot} \vec{H}_{t}\right\|^{2},
\end{array}
$$




$$
\begin{aligned}
& z(t)=\sqrt{\rho}\left\|\vec{f} g_{t}\right\|+\frac{1}{\sqrt{\sigma}}\left\|\xi_{t} \operatorname{rot} \vec{j}\right\|, \\
& I(t)=\int_{\Omega}\left[\vec{v}_{x_{k}}\left(\mu H_{k t} \vec{H}_{t}-\rho v_{k t} \vec{v}_{t}\right)+\mu \vec{H}_{x_{k}}\left(H_{k t} \vec{v}_{t}-v_{k t} \vec{H}_{t}\right)\right] d x .
\end{aligned}
$$

By applying the Young inequality to the right-hand side of (59), we obtain

$$
\begin{aligned}
&|I(t)| \leq\left(\int_{\Omega}\left(\mu H_{k t} \vec{H}_{t}+\rho v_{k t} \vec{v}_{t}\right)^{2}\right)^{\frac{1}{2}}\left\|\vec{v}_{x}\right\|+\mu \int_{\Omega} 2 \vec{H}_{x_{k}} \vec{H}_{t} \vec{v}_{t} d x \\
& \leq c_{3}\left(\int_{\Omega}\left(\left(\vec{H}_{t}^{2}\right)^{2}+\left(\vec{v}_{t}^{2}\right)^{2}\right) d x\right)^{\frac{1}{2}}\left\|\vec{v}_{x}\right\|+2 \mu\left(\int_{\Omega} \vec{H}_{t}^{2} \cdot \vec{v}_{t}^{2} d x\right)^{\frac{1}{2}}\left\|\vec{H}_{x}\right\| \\
& \leq c_{3} \Phi(t) \omega(t) F(t), \\
&\left|\rho \int_{\Omega} \vec{f} g_{t} \cdot \vec{v}_{t} d x+\frac{1}{\sigma} \int_{\Omega} \vec{H}_{t} \cdot \xi_{t} \operatorname{rot} \vec{j} d x\right| \\
& \leq \rho\left\|\vec{f} g_{t}\right\|\left\|\vec{v}_{t}\right\|+\frac{1}{\sigma}\left\|\xi_{t} \operatorname{rot} \vec{j}\right\|\left\|\vec{H}_{t}\right\| \\
& \leq \omega(t)\left(\sqrt{\rho}\left\|\vec{f} g_{t}\right\|+\frac{1}{\sqrt{\sigma}}\left\|\xi_{t} \operatorname{rot} \vec{j}\right\|\right) .
\end{aligned}
$$

Then we have

$$
\begin{aligned}
\frac{1}{2} \frac{d \omega^{2}(t)}{d t}+F^{2}(t) & \leq c \Phi(t) \omega(t) F(t)+\omega(t) z(t) \\
& \leq \frac{\delta}{2} F^{2}(t)+\frac{c^{2}}{2 \delta} \Phi^{2}(t) \omega^{2}(t)+\omega(t) z(t), \\
\frac{d \omega(t)}{d t}+\frac{2-\delta}{2 d^{2}} \omega(t) & \leq \frac{c^{2}}{2 \delta} \Phi^{2}(t) \omega(t)+z(t) .
\end{aligned}
$$

Multiplying the last inequality by $\exp \{-\beta(T-t)\}, \beta=\frac{2-\delta}{2 d^{2}}$, we obtain

$$
e^{-\beta(T-t)} \frac{d \omega(t)}{d t}+\beta \omega(t) e^{-\beta(T-t)} \leq \frac{c^{2}}{2 \delta} \Phi^{2}(t) \omega(t) e^{-\beta(T-t)}+z(t) e^{-\beta(T-t)},
$$

here $y(t)=\omega(t) e^{-\beta(T-t)}, \alpha_{1}(t)=\frac{c^{2}}{2 \delta} \Phi^{2}(t), \alpha_{2}(t)=z(t) e^{-\beta(T-t)}$. Then the last inequality can be rewritten as

$$
\frac{d y(t)}{d t} \leq \alpha_{1}(t) y(t)+\alpha_{2}(t)
$$

By the Gronwall lemma, we have

$$
\begin{aligned}
& \exp \left\{-\int_{0}^{t} \alpha_{1}(\tau) d \tau\right\} \frac{d y(t)}{d t} \\
& \quad \leq \alpha_{1}(t) y(t) \exp \left\{-\int_{0}^{t} \alpha_{1}(\tau) d \tau\right\}+\alpha_{2}(t) \exp \left\{-\int_{0}^{t} \alpha_{1}(\tau) d \tau\right\}, \\
& \frac{d}{d t}\left[y(t) e^{-\int_{0}^{t} \alpha_{1}(\tau) d \tau}\right] \leq \alpha_{2}(t) e^{-\int_{0}^{t} \alpha_{1}(\tau) d \tau} .
\end{aligned}
$$


Integrating this inequality with respect to $t$ from 0 to $T$, we obtain

$$
\begin{aligned}
& y(T) e^{-\int_{0}^{T} \alpha_{1}(\tau) d \tau} \leq y(0)+\int_{0}^{T} \alpha_{2}(t) e^{-\int_{0}^{t} \alpha_{1}(\tau) d \tau} d t, \\
& \omega(T) \leq \exp \left\{\frac{c^{2}}{2 \delta} \int_{0}^{T} \Phi^{2}(t) d t\right\}\left[\omega(0) e^{-\beta T}+\int_{0}^{T} z(t) e^{-\beta(T-t)} d t\right] .
\end{aligned}
$$

Returning to the original notation, we obtain the following inequality:

$$
\begin{aligned}
\rho\left\|\vec{v}_{t}(x, T)\right\|^{2}+\mu\left\|\vec{H}_{t}(x, T)\right\|^{2} \\
\leq \exp \left\{\frac{c^{2}}{2 \delta} \int_{0}^{T} \Phi^{2}(t) d t\right\}\left[\left(\rho\left\|\vec{v}_{t}(x, 0)\right\|^{2}+\mu\left\|\vec{H}_{t}(x, 0)\right\|^{2}\right) e^{-\frac{2-\delta}{2 d^{2}} T}\right. \\
\left.\quad+\int_{0}^{T}\left(\sqrt{\rho}\left\|g_{t}(x, t) \vec{f}(x)\right\|+\frac{1}{\sqrt{\sigma}}\left\|\xi_{t}(x, t) \operatorname{rot} \vec{j}(x)\right\|\right) e^{-\frac{2-\delta}{2 d^{2}}(T-t)} d t\right],
\end{aligned}
$$

where $\vec{v}_{t}(x, 0)=v \Delta \vec{v}_{0}+\vec{f}(x) g(x, 0)-v_{0 k} \vec{v}_{0 x_{k}}+\frac{\mu}{\rho} H_{0 k} \vec{H}_{0 x_{k}}$,

$$
\vec{H}_{t}(x, 0)=\frac{1}{\sigma \mu} \operatorname{rot} \operatorname{rot} \vec{H}_{0}+\frac{\xi(x, 0)}{\sigma \mu} \operatorname{rot} \vec{j}(x)+\operatorname{rot}\left[\vec{v}_{0} \times \vec{H}_{0}\right]
$$

We consider the bounded, convex, closed set

$$
D=\left\{\vec{f} \in L_{2}(\Omega), \operatorname{rot} \vec{j} \in L_{2}(\Omega),\|\vec{f}\| \leq 1,\|\vec{r}\| \leq 1\right\} .
$$

Since $(A \vec{f})(x)=\frac{1}{g(x, T)}\left(T_{g} \vec{f}\right)(x),(B \vec{r})(x)=\frac{\sigma \mu}{\xi(x, T)}\left(S_{\xi} \vec{r}\right)(x)$, the following inequality holds:

$$
\|A \vec{f}\|^{2}+\|B \vec{r}\|^{2} \leq M_{1}^{2}
$$

for the operators $A$ and $B$ in $D$.

We define in $D$ the nonlinear operators $A_{1}$ and $B_{1}$ in the following form:

$$
A_{1} \vec{f}=A \vec{f}+\vec{\kappa}, \quad B_{1} \vec{r}=B \vec{r}+\vec{\lambda}
$$

By the condition (53) the operators $A_{1}$ and $B_{1}$ map $D$ onto itself. By virtue of this and by Theorem 3, the operators $A$ and $B$ are completely continuous, and the combined Schauder principle implies the solvability of the operator equation

$$
A_{1} \vec{f}=\vec{f}, \quad B_{1} \vec{r}=\vec{r}
$$

in $D$. Thus, by Theorem 4 the inverse problem is solvable (1)-(6). The proof is complete.

\section{Conclusion}

The inverse problem with final overdetermination for a non-stationary magnetic hydrodynamics system has been reduced to an operator equation. By skillfully using the method proposed in [21] and [30], the compactness of the operator is proved and Schauder's theorem for the operator equation is used. The important thing is the previously unexamined 
inverse problem with final overdetermination for a non-stationary flat system of magnetic hydrodynamics. The results were formulated in the form of theorems and were proved rigorously. The search for new methods for facilitating the solution of the problem of the existence of a global solution of inverse problems for the Navier-Stokes equations, free convection, magnetohydrodynamics, and other nonlinear evolution equations is relevant. Therefore the proposed method by [21] and [30] is definitely applicable, also to the investigation of many other inverse problems.

Competing interests

The authors declare that they have no competing interests.

Authors' contributions

All authors contributed to the writing of this article equally. All authors read and approved the final manuscript.

\section{Acknowledgements}

This work was supported by the research project MES RK (No. 0113RK00943).

Received: 15 December 2014 Accepted: 7 September 2015 Published online: 23 September 2015

\section{References}

1. Leray, J: Etude de diverses equatiuos, integrales non lineaire et de quelques problemes que posent I'hydrodynamique. J. Math. Pures Appl. 35(12), 1-82 (1933)

2. Leray, J: Sur le mouvement d'un liquide visqueux emplissant l'espace. Acta Math. 63, 193-248 (1934)

3. Leray, J: Essai sur les mouvements peans d'un liquide visqueux que limitend des porous. J. Math. Pures Appl. 13, 331-418 (1934)

4. Ladyzhenskaya, OA: The Mathematical Theory of Viscous Incompressible Flow. Gordon \& Breach, New York (1996)

5. Temam, R: Navier-Stokes Equations: Theory and Numerical Analysis. North-Holland, Amsterdam (1977)

6. Lions, J-L: Some Methods for Solving Nonlinear Boundary Problems. Mir, Moscow (1972) (in Russian)

7. Antontsev, SN, Kazhikhov, AV, Monakhov, VN: Boundary Value Problems in Mechanics of Nonhomogeneous Fluids. North-Holland, Amsterdam (1990)

8. Solonnikov, VA: Estimates for solutions of nonstationary Navier-Stokes equations. Zap. Nauč. Semin. LOMI Steklova 38, 153-231 (1973) (in Russian); English transl. in J. Sov. Math. 8(4), 467-529 (1977)

9. Ladyzhenskaya, OA, Solonnikov, VA: Solution of some non-stationary problems of magnetohydrodynamics for a viscous incompressible fluid. Tr. Mat. Inst. Steklova 59, 115-173 (1960) (in Russian)

10. Ladyzhenskaya, OA, Solonnikov, VA: Some stationary boundary value problems of magnetohydrodynamics. Tr. Mat. Inst. Steklova 59, 174-187 (1960) (in Russian)

11. Mosconi, S, Solonnikov, VA: On a problem of magnetohydrodynamics in a multiconnected domain. Nonlinear Anal. 74(2), 462-478 (2011)

12. Ladyzhenskaya, OA, Solonnikov, VA: The linearization principle and invariant manifolds for problems of magnetohydrodynamics. Zap. Nauč. Semin. LOMI Steklova 38, 46-93 (1973) (in Russian); English transl. in J. Sov. Math. 8(4), 384-422 (1977)

13. Sakhaev, S, Solonnikov, VA: Estimates for solutions of a boundary value problem in magnetohydrodynamics. Tr. Mat. Inst. Steklova 127, 76-92 (1975) (in Russian); English transl. in Proc. Steklov Inst. Math. 127, 87-108 (1975)

14. Stupyalis, LI: The solvability of the initial boundary-value problem for magnetohydrodynamics. Zap. Nauč. Semin. LOMI Steklova 69, $219-239$ (1977) (in Russian); English transl. in J. Sov. Math. 10(1), 155-170 (1978)

15. Stupyalis, LI: A nonstationary problem of magnetohydrodynamics for the case of two space variables. Tr. Mat. Inst. Steklova 147(10), 156-168 (1980) (in Russian); English transl. in Proc. Steklov Inst. Math. 147, 163-175 (1981)

16. Alekseev, GV, Tereshko, DA: Analysis and Optimization in Viscous Fluid Dynamics. Dal'nauka, Vladivostok (2008) (in Russian)

17. Duvant, G, Lions, J-L: Inequations en thermoelasticite et magnetohydrodynamique. Arch. Ration. Mech. Anal. 48, 241-279 (1973)

18. Sermange, M, Temam, R: Some mathematical questions related to the MHD equations. Commun. Pure Appl. Math. 36, 635-664 (1983)

19. Giga, Y, Yoshida, Z: On the equations of the two-component theory in magnetohydrodynamics. Commun. Partial Differ. Equ. 9(6), 503-522 (1984)

20. Dyer, RH, Edmunds, DE: On the existence of solutions of the equations of magnetohydrodynamics. Arch. Ration. Mech. Anal. 9, 403-410 (1962)

21. Prilepko, Al, Vasin, IA: Inverse initial boundary value problems for nonstationary linearized Navier-Stokes equations. Differ. Equ. 25(1), 106-117 (1989) (in Russian)

22. Abylkairov, UU: The inverse problem for the Navier-Stokes. In: 7th Czechoslovak Conference on Differential Equations and Their Application (EQADIFF7), Praha, pp. 1-2 (1989)

23. Prilepko, AI, Vasin, IA: The solvability of the three-dimensional inverse problem for the non-linear Navier-Stokes equations. Zh. Vychisl. Mat. Mat. Fiz. 30(10), 1540-1552 (1990) (in Russian); English transl. in USSR Comput. Math. Math. Phys. 30(5), 189-199 (1990)

24. Prilepko, Al, Vasin, IA: On a nonlinear nonstationary inverse problem of hydrodynamics. Inverse Probl. 7(2), 13-16 (1991) 
25. Vasin, IA: The existence and uniqueness of the generalized solution of the inverse problem for the nonlinear nonstationary Navier-Stokes system in the case of integral overdetermination. Mat. Zametki 54(4), 34-44 (1993) (in Russian); English transl. in Math. Notes 54(3-4), 1002-1009 (1993)

26. Prilepko, Al, Orlovsky, DG, Vasin, IA: Method for Solving Inverse Problems in Mathematical Physics. Dekker, New York (2000)

27. Fan, J, Nakamura, G: Well-posedness of an inverse problem of Navier-Stokes equations with the final overdetermination. J. Inverse III-Posed Probl. 17(6), 565-584 (2009)

28. Choulli, M, Imanuvilov, OY, Puel, J-P, Yamamoto, M: Inverse source problem for the linearized Navier-Stokes equations with interior data in arbitrary sub-domain. Appl. Anal. 92, 2127-2143 (2013)

29. Chebotarev, AY: Subdifferential inverse problems for evolution Navier-Stokes systems. J. Inverse III-Posed Probl. 8(3), 243-254 (2013)

30. Abylkairov, UU: Unique solvability of the problem flow for 2D-3D Navier-Stokes. Mat. Zh. Respub. Kaz. 5(3), 11-18 (2005) (in Russian)

31. Lions, J-L: Optimal Control of Systems Governed by Partial Differential Equations. Springer, Berlin (1972)

32. Fursikov, AV: Optimal Control of Distributed Systems: Theory and Applications. Translations of Mathematical Monographs. Nauchnaya Kniga, Novosibirsk (1999) (in Russian); English edition: Am. Math. Soc. (2000)

33. Chebotarev, AY: Subdifferential inverse problems for magnetohydrodynamics. Methods Appl. Anal. 17(4), 395-406 (2010)

34. Chebotarev, AY: Determination of the right-hand side of the Navier-Stokes system of equations and inverse problems for the thermal convection equations. Comput. Math. Math. Phys. 51(12), 2146-2154 (2011)

35. Chebotarev, AY: Inverse problem for Navier-Stokes systems with finite-dimensional overdetermination. J. Differ. Equ 48(8), 1153-1160 (2012)

\section{Submit your manuscript to a SpringerOpen ${ }^{\circ}$ journal and benefit from:}

- Convenient online submission

- Rigorous peer review

- Immediate publication on acceptance

- Open access: articles freely available online

High visibility within the field

Retaining the copyright to your article 